SNS Ring BPM

BNL/SNS TECHNICALL NOTE

NO. 069

S. Y. Zhang

December 15, 1999

COLLIDER ACCELERATOR DEPARTMENT

BROOKHAVEN NATIONAL LABORATORY

UPTON, NEW YORK 11973 


\title{
SNS Ring BPM
}

\author{
S.Y. Zhang
}

\begin{abstract}
The sensitivity and impedance of stripline and capacitive pickups are compared with respect to the SNS storage ring conditions. Transfer functions of these pickups are given. In general, the capacitive pickup is more sensitive, but the associated impedance is also higher than the stripline pickup. The low frequency impedance of the capacitive pickup has similarities with the wall resistance, but it does less harm in terms of beam instabilities, compared with the wall impedance.
\end{abstract}




\section{Introduction}

The use of the Beam Position Monitor (BPM) system for the Spallation Neutron Source (SNS) accumulator ring includes:

1. Closed orbit measurement and correction.

2. Tune measurement.

3. RF radial control.

4. Tracking for the painting.

5. Observe the transverse motion within the bunch.

On the other hand, the basic requirement for the BPM system includes,

1. Intensity from a quarter of the first turn injection, $5 \times 10^{10}$, to $2 \times 10^{14}$ protons per bunch.

2. Beam emittance up to $160 \pi \mu \mathrm{m}$, with additional range of $\pm 20 \mathrm{~mm}$.

3. Linearity consistent with the accuracy of $\pm 1 \mathrm{~mm}$.

4. Long term stability consistent with the accuracy.

5. Turn-by-turn resolution for tracking of painting and corrections.

6. Physical space less than $50 \mathrm{~cm}$ for each set of detector.

7. Reliability, radiation damage protection.

To decide which type of BPM, i.e. stripline or capacitive pick-up, will be used mainly serving these uses and satisfying the requirements, a comparison of these two type of detectors is presented in this note. Two main issues are discussed, i.e. the sensitivity and the impedance.

Under the condition of the SNS accumulator ring, in general, following results are discussed.

1. Stripline BPM essentially senses $d I_{b} / d t$, and capacitive pickup senses $I_{b}$. With the same charge in the bunch, the detected voltage at the capacitive pickup is proportional to $1 / \sigma$, whereas the stripline detector to $1 / \sigma^{2}$, where $\sigma$ is the $r m s$ bunch length. 
2. For the SNS long bunch of $550 \mathrm{~ns}$, the capacitive pickup is, in general, about 20 to 40 times more sensitive than the stripline BPM. Under the same condition, therefore, the sensitivity of both types of pickups is similar if the bunch is $12 n s$ long. Also, for $200 \mathrm{MHz}$ Linac microbunches, the stripline is about three times as sensitive as the capacitive pickup.

3. Taking into consideration of the bleeder resistance $R_{b}$, the sensitivity of the capacitive pickup is reduced. The voltage of the capacitive pickup with small $R_{b} C$ looks more like the one of stripline BPM, where $C$ is the plate to ground capacitance.

4. Impedance of stripline BPM is well understood, and it is relatively low.

5. The impedance of capacitive pickup involves three factors, 1 . the low frequency part determined by $R_{b}$ and $C, 2$. the resonant mode determined by the length of the plate, and 3. the termination, which affects significantly the resonant mode.

6. The low frequency impedance is more important, which is somewhat similar to the resistive wall impedance, but does less harm compared with the wall resistance.

7. The higher sensitivity of the capacitive pickup is associated with higher impedance, compared with the stripline detector. It is also true that for capacitive pickup, the impedance can be reduced by reducing the sensitivity.

\section{Sensitivity}

\section{A. Stripline pickup}

Consider the stripline BPM with the length $\ell$, the subtends $\phi_{0}$, and the characteristic impedance $Z_{c}$. Assuming the wave propagating velocity in the stripline is the same of the beam, $\beta c$, the net voltage at matched terminated upstream port is $[1,2]$,

$$
V_{s}(t)=\frac{\phi_{0}}{2 \pi} \frac{Z_{c}}{2}\left(I_{b}(t)-I_{b}\left(t-\frac{2 \ell}{\beta c}\right)\right)
$$


In other words, this voltage is proportional to the difference between the beam signal and the reflected current.

In frequency domain, this voltage is

$$
V_{s}(\omega)=\frac{\phi_{0}}{2 \pi} \frac{Z_{c}}{2} I_{b}(\omega)\left(1-\exp \left(-j \frac{2 \omega \ell}{\beta c}\right)\right)
$$

which will be used in calculating the impedance.

Equation 2 shows that under the condition of $2 \omega \ell / \beta c \ll 1$, the stripline pickups detect $d I_{b} / d t$. In fact, we have,

$$
V_{s}(\omega) \approx \frac{\phi_{0} Z_{c}}{2 \pi} I_{b}(\omega) \frac{j \omega \ell}{\beta c}
$$

For $\ell=25 \mathrm{~cm}$ of the SNS design, the equation 3 can be used for $f<$ $M H z$.

\section{B. Capacitive pickup}

For a capacitive pickup with the length $\ell$, the subtends $\dot{\phi}_{0}$, there are two more important parameters. These are the capacitance and the bleeder resistance between the plate and ground, $C$ and $R_{b}$. The total charge induced by the beam at the plate is [3],

$$
q=-\frac{\phi_{0}}{2 \pi} \frac{\ell}{\beta c} I_{b}
$$

and the voltage developed across the resistance is

$$
V_{c}(\omega)=-j \omega q \frac{R_{b}}{1+j \omega R_{b} C}=\frac{\phi_{0}}{2 \pi} \frac{\ell}{\beta c} I_{b}(\omega) \frac{}{1+j \omega R_{b} C}
$$

This equation shows that the capacitive pickups senses the beam current $I_{b}$, with a transient response represented by $j \omega R_{b} /\left(1+j \omega R_{b} C\right)$.

It is not straightforward to get the voltage in time domain, because it depends on the particular beam current $I_{b}$. However, assuming $\omega R_{b} C>1$, we can get [3],

where $V_{c 0}$ is the offset voltage. 


\section{Comparison}

Since the stripline essentially senses $d I_{b} / d t$, and capacitive senses $I_{b}$, for very long bunch of $\sim 550 n s$ at the SNS ring, the capacitive pickup is more sensitive. To have a comparison, we set up the following parameters. The stripline has $\ell=25 \mathrm{~cm}, \phi_{0}=70^{\circ}$, and $Z_{c}=50 \Omega$, and capacitive has $\ell=25$ $\mathrm{cm}, \phi_{0}=140^{\circ}, C=200 \mathrm{pf}$. Consider a Gaussian beam line density with $r m s$ length of $\sigma=137.5 \mathrm{~ns}$, or total length of $550 \mathrm{~ns}$, and with charges of $2 \times 10^{11}$ protons in a bunch. The voltage of stripline and capacitive pickups is shown in Fig.1, where equations 1 and 5 are used. Note that in this case the peak voltage of the capacitive and stripline pickups is $180 \mathrm{mV}$ and \pm 4 $m V$, respectively.

With the same charge in the bunch, the detected voltage at the capacitive pickup is proportional to $1 / \sigma$, whereas the stripline detector to $1 / \sigma^{2}$. Therefore, for a bunch with the total length of $12 n s$, two type of pickups give rise to similar peak voltage. Also, for $200 \mathrm{MHz}$ Linac microbunches, with the bunch length of $2 n s$, the stripline is about three times more sensitive than capacitive pickup. Note that under this condition, the pickup voltage pattern is more complicated than $d I_{b} / d t$.

Another example is that for RHIC, the most concerned case is the Gold beam at the injection. Using the stripline length of $20 \mathrm{~cm}$, for $20 \mathrm{~ns}$ long $A u^{79+}$ ions of $10^{9}$ a bunch, the BPM voltage is at the comfortable $\pm 0.8 \mathrm{~V}$, for Gaussian beam line density.

Note that the capacitance between the plate and ground is important to determine the detected voltage. Smaller capacitance gives rise,to higher voltage. For the AGS Booster capacitive pickup, $C \approx 200 p f$ for high gain mode, where about $3 / 4$ of the capacitance comes from the cable [4]. A measurement shows that for the AGS capacitive pickup, which is 4-piece split, this capacitance is $\sim 50 p f$ [5].

Note also in this comparison, the bleeder resistance of the capacitive pickup is not included. Consider the factor of $j \omega R_{b} /\left(1+j \omega R_{b} C\right)$. If $\omega R_{b} C>$ 1 , the pickup senses the beam current, which can be observed from equation 5. Otherwise, if $\omega R_{b} C<1$, the pickup looks more like to sense $d I_{b} / d t$, which can be observed from the following equation, obtained under this condition,

$$
V_{c}(\omega) \approx \frac{\phi_{0}}{2 \pi} \frac{\ell}{\beta c} I_{b}(\omega) j \omega R_{b}
$$

This equation is identical to equation 3 , if letting $Z_{c}=R_{b}$. 
In the SNS ring, the revolution frequency is $1.189 \mathrm{MHz}$. If we take $C=200 p f$, then the critical bleeder resistance is $R_{b}=670 \Omega$. The resistance smaller than $670 \Omega$ will let the capacitive pickup to sense $d I_{b} / d t$, rather than $I_{b}$.

In Fig. 2a, the first 10 turns of injection is shown with the sine beam line density. The parameters used for two types of pickups are the same as that in Fig. 1. In Fig. 2b, the stripline pickup voltage is shown. The largest voltage for the first turn is $4.9 \mathrm{mV}$, which is a little higher than that in Fig. 1 , because of the different beam line density. In Fig. 2c, The capacitive voltage is shown, which is obtained by simulation, rather than the steady state of equation 6 . The bleeder resistance $R_{b}=500 \Omega$ is used, and the peak voltage detected for the first turn is $64 \mathrm{mV}$, which is lower than 180 $m V$ in Fig.1. Note that this resistance is lower than the critical resistance of $R_{b}=670 \Omega$. In Fig. 2d, $R_{b}=50 \Omega$ is used, the voltage looks like the one from the stripline, and the peak voltage for the first turn is further reduced to $9.5 \mathrm{mV}$.

Taking $R_{b}>670 \Omega$, say $2 k \Omega$, the beam line density is detected directly, and the peak voltage for the first turn is increased to $120 \mathrm{mV}$.

\section{Impedance}

\section{A. Stripline pickup}

Consider the stripline BPM. Using equation 2, it is straightforward to get the longitudinal impedance for one strip plate $[1,2]$,

$$
Z_{s \ell}=Z_{c}\left(\frac{\phi_{0}}{2 \pi}\right)^{2}\left(\sin ^{2} \frac{\omega \ell}{\beta c}+j \sin \frac{\omega \ell}{\beta c} \cos \frac{\omega \ell}{\beta c}\right)
$$

One may notice that the lowest resonant frequency of the impedance is at $\beta c /(4 \ell)$. For frequency much smaller than $\beta c /(4 \ell)$, the reactive part can be estimated as,

$$
\left(\frac{Z_{s \ell}}{n}\right)_{i m a g}=j \frac{Z_{c}}{n}\left(\frac{\phi_{0}}{2 \pi}\right)^{2} \sin \frac{\omega \ell}{\beta c} \cos \frac{\omega \ell}{\beta c} \approx j Z_{c}\left(\frac{\phi_{0}}{2 \pi}\right)^{2} \frac{\ell}{R}
$$


The real part is small at the low frequency, and it reaches maximum at $f=\beta c /(4 \ell)$,

$$
\left(\frac{Z_{s \ell}}{n}\right)_{\text {real }, \max }=\left(\frac{Z_{c}}{n}\left(\frac{\phi_{0}}{2 \pi}\right)^{2} \sin ^{2} \frac{\omega \ell}{\beta c}\right)_{\max } \approx Z_{c}\left(\frac{\phi_{0}}{2 \pi}\right)^{2} \frac{2 \ell}{\pi R}
$$

The transverse impedance is obtained using Nassibian-Sacherer derivations [6], for a pair of striplines,

$$
Z_{s T}=\frac{R}{\beta b^{2}}\left(\frac{4}{\phi_{0}}\right)^{2} \sin ^{2}\left(\frac{\phi_{0}}{2}\right)\left(\frac{Z_{s \ell}}{n}\right)
$$

\section{B. Capacitive pickup}

The impedance of capacitive pickup involves three aspects,

1. Low frequency part determined by $R_{b}$ and $C$ [7].

2. Resonant mode determined by the length of the plate.

3. The termination affects significantly the resonant mode.

For the low frequency part determined by $R_{b}$ and $C$, we use equation 5 to get the longitudinal impedance for one plate,

$$
Z_{c \ell}=\left(\frac{\phi_{0}}{2 \pi}\right)^{2} \frac{\ell}{\beta c} \frac{j \omega R_{b}}{1+j \omega R_{b} C}
$$

or

$$
\frac{Z_{c \ell}}{n}=\left(\frac{\phi_{0}}{2 \pi}\right)^{2} \frac{\ell}{R} \frac{j R_{b}}{1+j \omega R_{b} C}
$$

The transverse impedance is obtained similarly as,

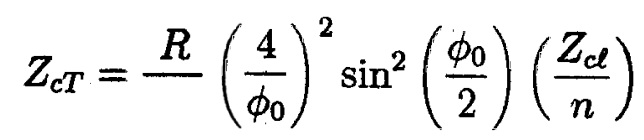

The comparison of impedance of stripline and capacitive pickup is shown in Fig. 3, where the same conditions in Fig. 2 is used. The longitudinal 
impedance of the stripline is for total 44 sets of detectors, each with 4 plates, and the transverse is for each direction. The impedance of capacitive pickups is also for 44 sets of detectors, the bleeder resistance is, however, taken as $500 \Omega$ and $50 \Omega$ for comparison. The resonant mode of the capacitive pickup impedance is determined by $R_{b} C$. Since we have $C=200 p f$, the resonant modes for $R_{b}=500 \Omega$ and $R_{b}=50 \Omega$ are at $1.6 \mathrm{MHz}$ and $16 \mathrm{MHz}$, respectively. The maximum real parts of the capacitive pickup with $R_{b}=500$ $\Omega$ and $R_{b}=50 \Omega$ are about 20 and 2 times of the stripline. Meanwhile, we recall that the sensitivities in these two cases are about 15 and 2 times higher than the stripline, see Fig. 2. Therefore, it looks that the higher sensitivity of capacitive pickup is paid by the larger impedance, which is not a surprise.

Equations 5 and 12 show that for capacitive pickup, the sensitivity is proportional to $\phi_{0} \ell$, and the impedance is proportional to $\phi_{0}^{2} \ell$. Given sensitivity, smaller subtends and longer length will give rise to smaller impedance.

The low frequency impedance of capacitive pickup carries some similarity with the resistive wall impedance. In Fig. 4, for 44 pair capacitive pickups, with the parameters as $\ell=10 \mathrm{~cm}, \phi_{0}=140^{\circ}, C=10 \mathrm{nf}$, and $R_{b}=500$ $\Omega$, the longitudinal impedance $Z_{c \ell} / n$ is shown in comparison with the SNS ring resistive wall. The real and imaginary part of the capacitive pickup are approximately proportional to $1 / \omega$ and $1 / \omega^{2}$, respectively. On the other hand, both real and imaginary parts of the resistive wall impedance are proportional to $1 / \sqrt{\omega}$.

The impact of the capacitive impedance on the beam instability is less severe compared with the resistive wall resistance. This is demonstrated in Fig. 5, where the SNS bunch spectrum and the real part of the wall and capacitive pickup impedance are shown. The sampling frequencies are shown by circles. If the sum of the impedance weighted by the spectrum at sampling points is negative, then the beam is unstable, otherwise, it is stable. For the resistive wall case, the sampling point at $-0.24 \mathrm{MHz}$, for the fractional tune of 0.8 , carries large amplitude of both impedance and spectrum, therefore, it is dominant. Since it is negative, the beam is unstable.

On the other hand, for the capacitive pickup impedance, in addition to $-0.24 \mathrm{MHz}$, two more sampling points involved, i.e. at $-1.43 \mathrm{MHz}$ and $0.95 \mathrm{MHz}$. In fact, the point at $0.95 \mathrm{MHz}$ has peak impedance and half spectrum amplitude, therefore, it is dominant. Since this is positive, the beam is stable. These results are obtained for zero chromaticity. 
For high frequency resonance, the measurement of Ratti and Shea [8] has shown that for the AGS Booster capacitive pickup, a resonance at $360 \mathrm{M} \mathrm{Hz}$ has the real part of impedance of $300 \Omega$, which corresponds to $Z_{c \ell} / n \approx 1 \Omega$. Total 48 pairs of capacitive pickup of the Booster would have $Z_{c \ell} / n_{\text {real, } \max } \approx$ $48 \Omega$. However, with termination, the resonant frequency is reduced to 240 $M H z$, and the impedance is reduced by a factor of 15 , giving rise to total $Z_{c \ell} / n_{\text {real,max }} \approx 4 \Omega$. Further investigation of the terminations is needed, however, this has demonstrated that the resonance at high frequency is not likely to cause serious damage in terms of beam instability. 


\section{References}

${ }^{1}$ R.E. Shafer, IEEE Trans. NS, Vol.NS-32,No.5, p.1933, 1985

${ }^{2}$ K.Y. Ng, Particle Accelerators, Vol.23, p.93, 1988.

${ }^{3}$ R.E. Shafer, AIP Conf. Proc. 249, p. 601, 1992.

${ }^{4}$ D.J. Ciardullo, AGS Booster Tech. Note, No. 178, 1990.

${ }^{5} \mathrm{~L}$. Ahrens, private communication.

${ }^{6}$ G. Nassibian and F. Sacherer, Nucl. Inst. Meth. Vol.159, p21, 1979.

${ }^{7}$ G. Lambertson, AIP Conf. Proc. 153, p. 1413, 1987.

${ }^{8}$ A. Ratti and T.J. Shea, p.1803, PAC 1991 

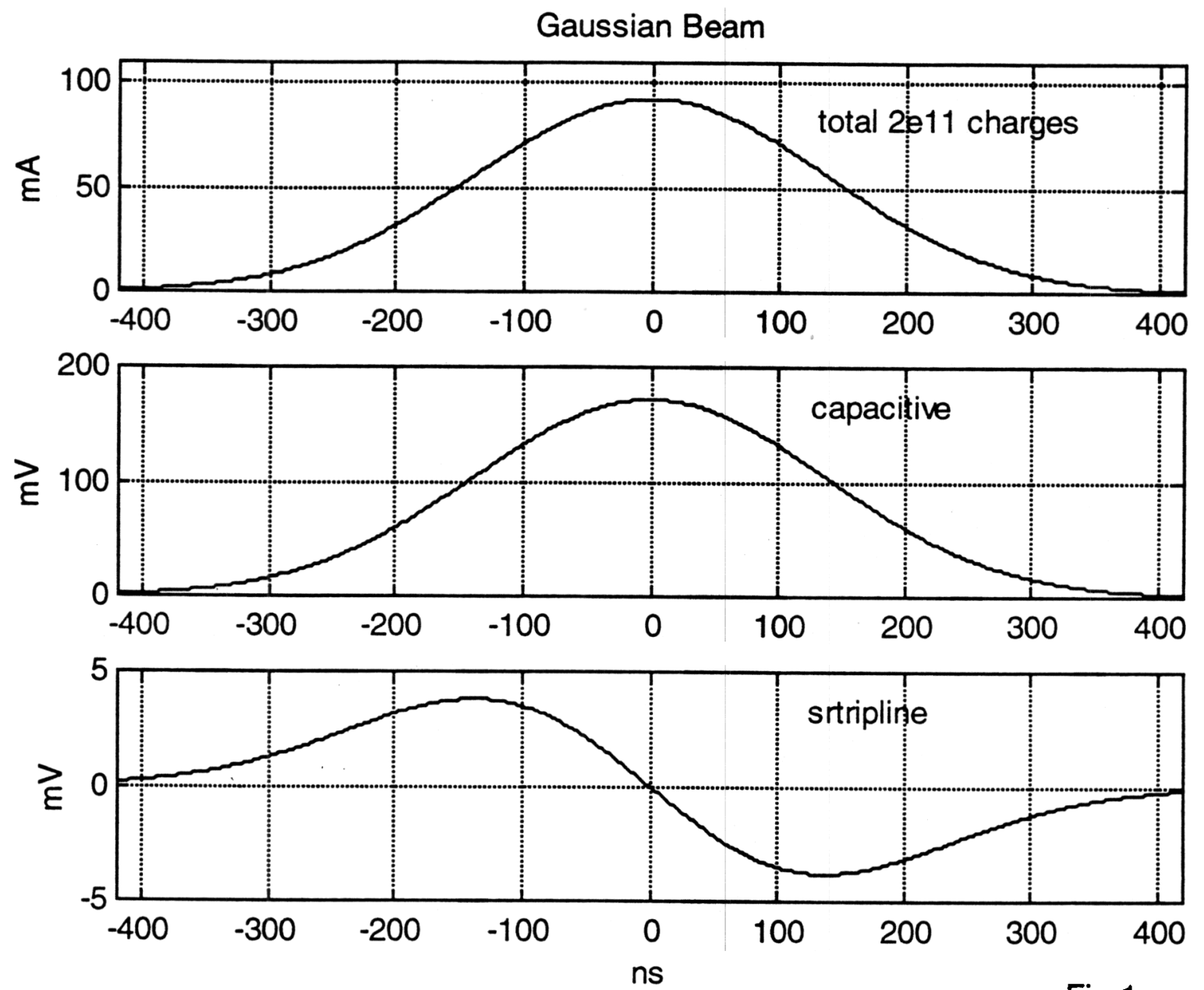

Fig.1

Fig.1

Sensitivity of capacitive and stripline pickups for Gaussian beam line density, total charge equals to first turn injection. 
First 10 tunrs of injection

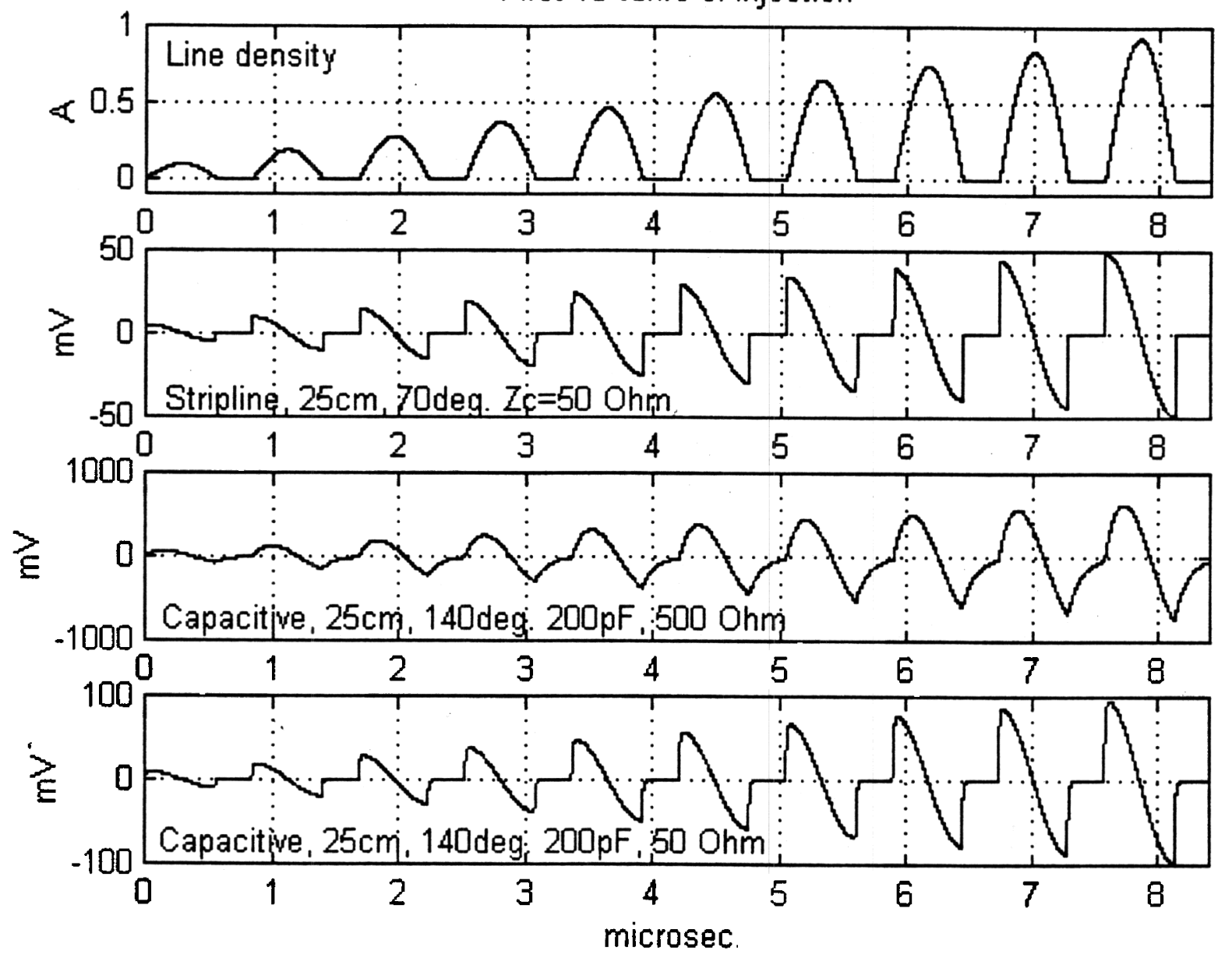

Fig.2

Comparison of sensitivity of stripline and capacitive pickups for the first 10 turns of injection.

Capacitive pickup bleeder resistance 500 and $50 \mathrm{Ohms}$. 

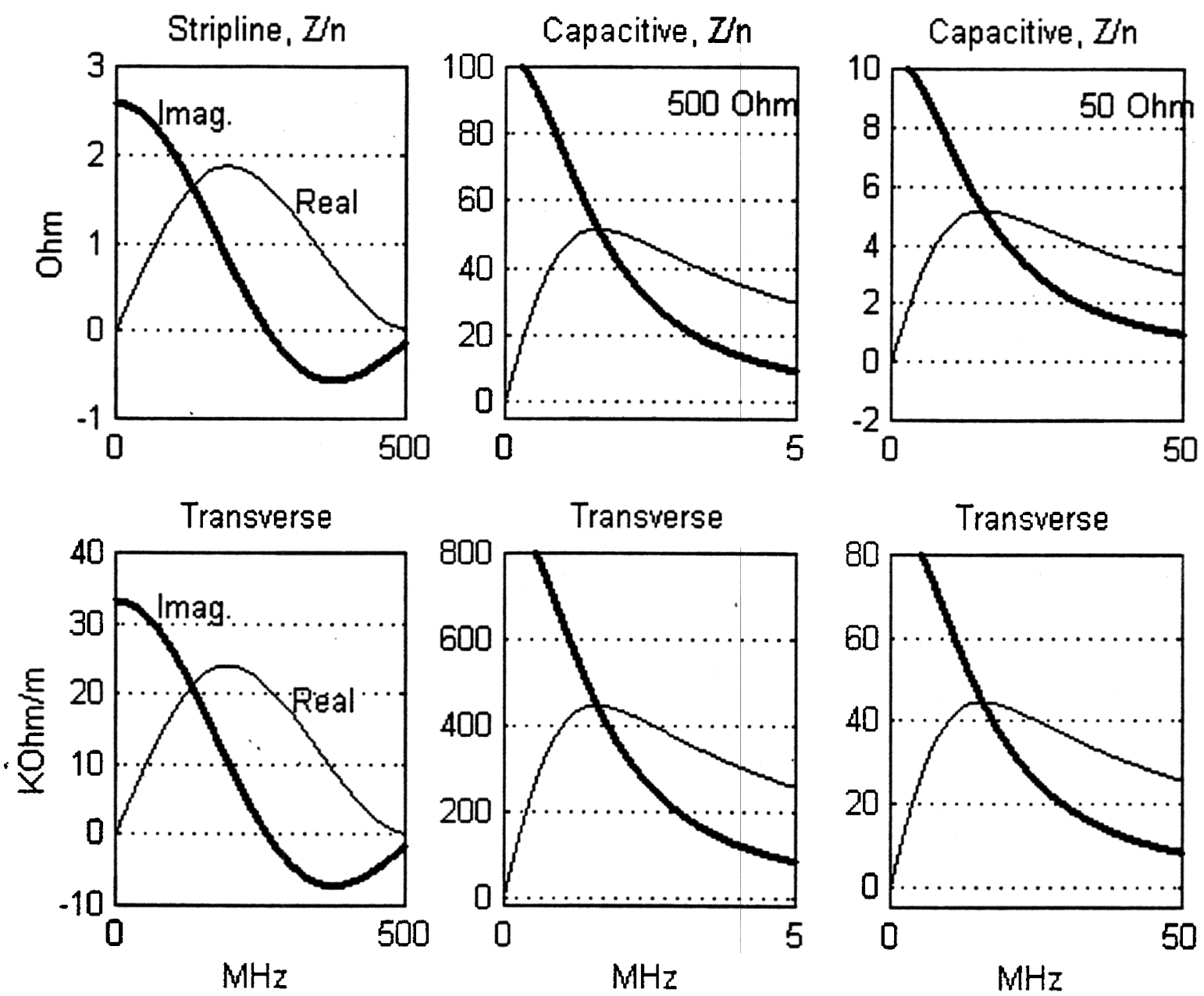

Fig. 3

Comparison of impedance.

Stripline: $25 \mathrm{~cm}, 70 \mathrm{deg}$., $\mathrm{Zc}=50 \mathrm{Ohm}$. 44 sets in SNS ring. Capacitive: $25 \mathrm{~cm}, 140$ deg., 200 pf, 44 pairs, 500 and 50 Ohms. 

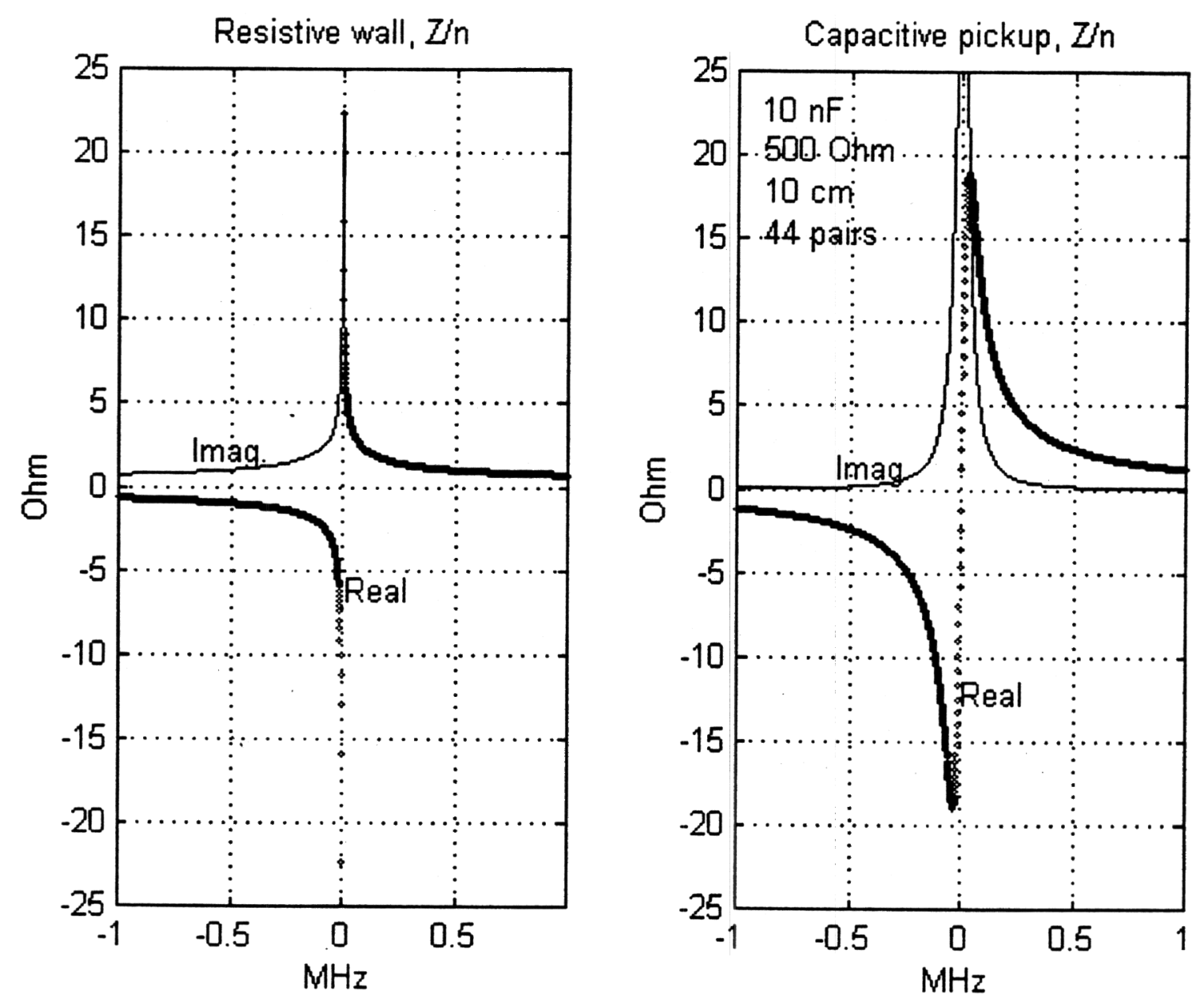

Fig. 4

Similarity of resistive wall and capacitive pickup impedances. 

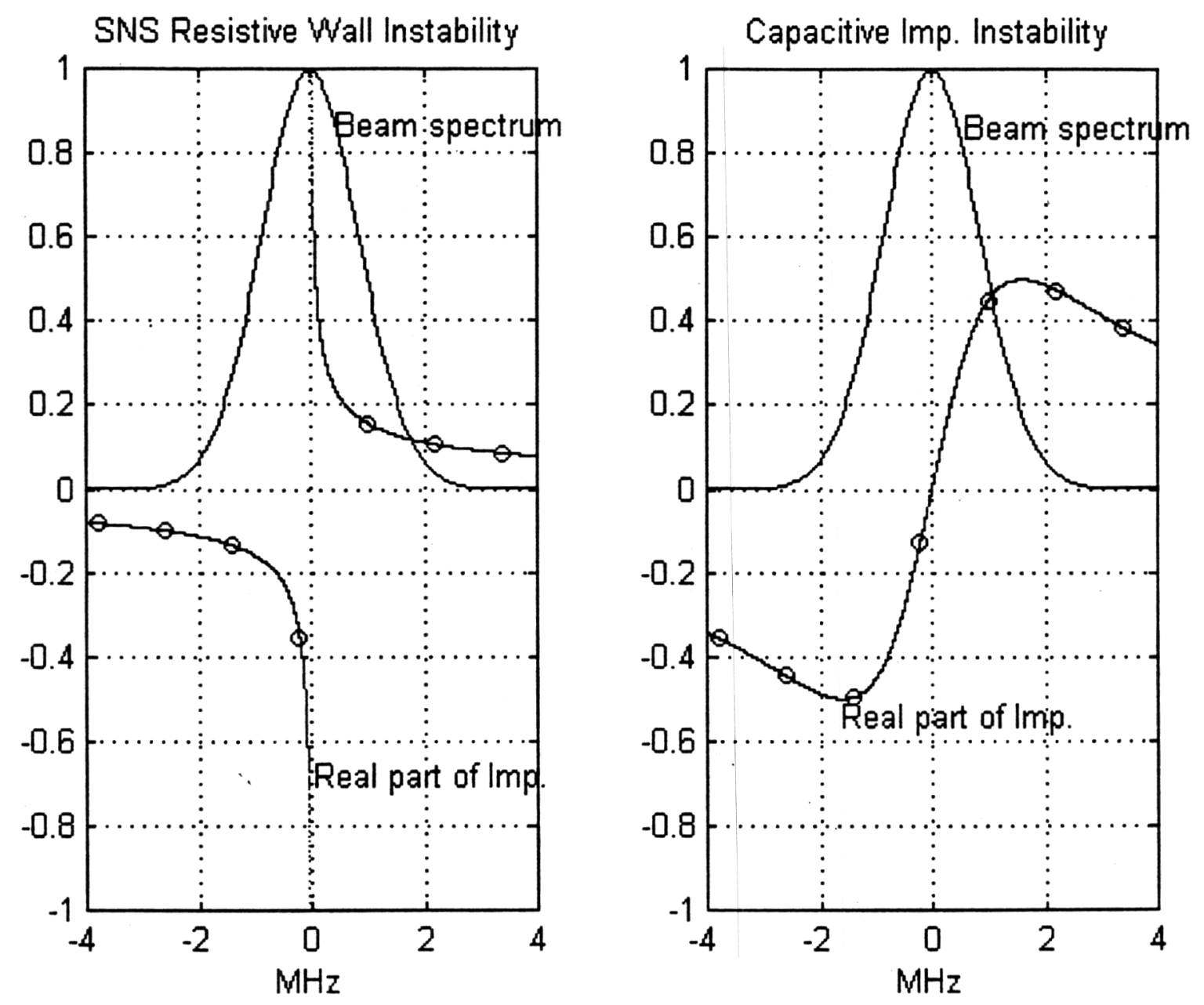

Fig. 5

Comparison of resistive wall and capacitive impedance with respect to beam instability. 